\title{
Editorial
}

\section{Artificial Intelligence Applications in Civil Engineering}

\author{
Tayfun Dede $\mathbb{D}^{1}{ }^{1}$ Murat Kankal, ${ }^{2}$ Ali Reza Vosoughi, ${ }^{3}$ Maksym Grzywiński, ${ }^{4}$ \\ and Moacir Kripka ${ }^{5}{ }^{5}$
}

${ }^{1}$ Department of Civil Engineering, Karadeniz Technical University, Trabzon, Turkey

${ }^{2}$ Department of Civil Engineering, Uludăg University, Bursa, Turkey

${ }^{3}$ Department of Civil Engineering, Shiraz University, Shiraz, Iran

${ }^{4}$ Department of Civil Engineering, Czestochowa University of Technology, Czestochowa, Poland

${ }^{5}$ Department of Civil Engineering, Universidade de Passo Fundo, 99001-970 Passo Fundo, RS, Brazil

Correspondence should be addressed to Tayfun Dede; tayfundede@gmail.com

Received 9 April 2019; Accepted 10 April 2019; Published 2 May 2019

Copyright (C) 2019 Tayfun Dede et al. This is an open access article distributed under the Creative Commons Attribution License, which permits unrestricted use, distribution, and reproduction in any medium, provided the original work is properly cited.

With a great pleasure, we introduce this special edition of the Journal of Advances in Civil Engineering, the result of the joint effort of authors, editors, and reviewers.

Artificial intelligence is to develop the machine elements that analyze the human's thinking system and reflect the same to reality. In recent years, artificial intelligence applications have found a wide range of applications in civil engineering and the other engineering branches. The increase in artificial intelligence studies with great acceleration shows that the use of artificial intelligence in engineering branches will increase in the coming years.

The focus of this special issue is on applications of artificial intelligence approaches in civil engineering. Especially hybrid artificial intelligence studies in the fields of structural engineering, construction management, hydrology, hydraulic engineering, geotechnical engineering, environmental engineering, transportation engineering, coastal and ocean engineering, and materials of construction form the basis of this special issue. Besides, review articles including applications of civil engineering using branches of artificial intelligence techniques (ANN, fuzzy system, expert system, and swarm intelligence) is covered by this special issue.

During the call for submissions, more than 40 papers were received, focusing on several aspects related to the application of artificial intelligence in civil engineering. Twelve of these papers were accepted by the referee and the editor. This rigorous revision process can be seen as an indicative of the high quality of the accepted works. The details about the published papers are given in the sequence.

The first accepted paper prepared by Lu et al. presenting a risk assessment method for cable system construction of suspension bridges was based on the cloud model. The proposed model can combine randomness and fuzziness of risk information effectively. At the end of their study, the authors concluded that the risk assessment method can provide safety assurance and technical support for cable system construction of the long-span suspension bridge.

S. Petruseva et al. presented a hybrid method for predicting construction time in the early project phase. They used many ANN techniques such as General Regression Neural Network (GRNN), Backpropagation Neural Network (BNN), and Radial Base Function Neural Network (RBFNN). Their study shows that the BNN technique is better than the other techniques when they compared the results obtained. But, their developed model is not suitable for the higher, more intensive risk factors impact during the construction period.

H. Ceylan and T. Ozcan presented a case study on the optimization of headways and departure times in urban bus networks. The authors used the metaheuristic harmony search optimization method to evaluate the user and operator costs. This study gives Pareto solutions in terms of the user and operator benefits. At the end of the study, the authors concluded that total travel time and total service $\mathrm{km}$ could be reduced by $4.8 \%$ and $9.8 \%$, respectively, compared with the current bus network. 
E.-T. Lee and H.-C. Eun investigated damage detection methods using the stress or stiffness variation rate of the truss element before and after the damage. To predict damaged elements depending on complete and incomplete measurement, the authors of this study consider some methods such as the substructuring method, damage detection methods, and static-based and dynamic-based substructuring method. The detailed knowledge about these methods can be found from the paper submitted to this special issue.

G. B. Jumaa and A. R. Yousif proposed three prediction models by using ANN. They are ANN, gene expression programming (GEP), and nonlinear regression analysis (NLR). The authors used a large database including 269 shear test results and the genetic programming to predict the shear capacity of FRP-reinforced concrete beams without stirrups. Their parametric study indicated that the ANN model defines accurately the interaction of all parameters on shear capacity prediction.

C.-Y. Kao et al. develops a two-step computer-aided approach for pozzolanic concrete mix design. The first step is establishing a dataset of pozzolanic concrete mixture proportioning which conforms to American Concrete Institute Code. In the first step, ANNs are employed to establish the prediction models of compressive strength and the slump of the concrete. Sensitivity analysis of the ANN is used to evaluate the effect of inputs on the output of the ANN. The two ANN models are tested using data of experimental specimens made in a laboratory for twelve different mixtures. The second step is classifying the dataset of pozzolanic concrete mixture proportioning. A classification method is utilized to categorize the dataset into 360 classes based on compressive strength, pozzolanic admixture replacement rate, and material cost. Thus, one can easily obtain mix solutions based on these factors. The results show that the proposed computer-aided approach is convenient for pozzolanic concrete mix design and practical for engineering applications.

N.-D. Hoang's research establishes an automatic approach for asphalt pavement pothole detection. Image processing techniques including Gaussian filter (GF), steerable filter (SF), and integral projection (IP) are used synergistically to extract features from pavement digital images. Two levels of GF are utilized as an image denoising technique. SF assisted by GF is used to generate a pothole resilient map. IP analysis based on such map is performed to numerically present the feature of an image with the particular interest in pothole recognition. A simple moving average technique is put forward to reduce the number of the extracted features from 300 to 60 . Based on the image features, two artificial intelligence (AI) approaches of ANN and the least squares support vector machine (LS-SVM) have been employed to construct classification models to predict the existence of pothole on the pavement surface. Experimental results with a repeated subsampling procedure with 20 runs confirm that ANN and LS-SVM are capable AI methods for pothole detection. It is because the classification accuracy rates (CARs) of both methods are higher than $85 \%$ and the area under the curve (AUC) values surpass 0.9.
Moreover, LS-SVM has been identified as the better approach for the task of pothole detection with a desired accuracy of approximately $89 \%$.

S. Golnaraghi et al. used four different ANN methods: Backpropagation Neural Network (BNN), Radial Basis Network (RBF), Generalized Regression Neural Network (GRNN), and Adaptive Neuro-Fuzzy Inference System (ANFIS) to model labour productivity. Weather (temperature, humidity, wind speed, and precipitation), crew (gang size and labour percentage) and project (work type, floor level, and work method) data were selected as inputs in the models. According to $R^{2}$ values, the best result in the models was obtained from BNN with 0.98 for the training set and from RBF with 0.85 for the test set. In the three methods other than $\mathrm{RBF}$, it was determined that temperature was the most important parameter affecting labour productivity. It was determined that the best model $\mathrm{BNN}$ obtained as a result of this study can help save time and cost associated with quantifying loss of productivity.

J. A. Álvarez et al. predicted energy performance of a house using ANN models to evaluate building energy efficiency. The dataset obtained from 453 buildings located in the northern area of Spain with a total usable area of $570,438.30 \mathrm{~m}^{2}$ was used in the models. The data were divided into two: $90 \%$ of them were training set, and $10 \%$ of them were validation set. Together with this study, it is possible to estimate the energy efficiency of a building in a certain region by using some characteristics belonging to that building with high accuracy without interfering with the building or using any measuring device.

A. M. al-Swaidani and W. T. Khwies applied the ANN and multilinear regression (MLR) models to estimate 2, 7, 28, 90, and 180 days compressive strength, water permeability, and porosity of concretes containing volcanic scoria as cement replacement. Cement content, volcanic scoria content, water content, superplasticizer content, and curing time were used as model inputs. The data used in the ANN models were divided into $70 \%$ training, $15 \%$ testing, and $15 \%$ validation pattern, respectively. Sensitivity analysis showed that all parameters used as an input in this study have significant effects on the properties of concrete containing volcanic scoria as cement replacement. The results showed that ANN models were much more accurate than MLR models and that ANN can be used successfully to predict the investigated concrete properties.

$\mathrm{X}$. Cao et al. developed a new dynamic multicriteria decision-making approach for low-carbon supplier selection in low-carbon building construction projects. This approach includes interval-valued triangular fuzzy numbers intuitionistic fuzzy. According to the demand from the constructors during the considered projects, 5 main criteria and 17 subcriteria were established for the selection of lowcarbon suppliers in the construction sector. In conclusion, the authors revealed that the proposed model can be easily extended to analyze other management decision problems as a structural model.

N.-D. Hoang and Q.-L. Nguyen suggest a hybrid model that includes image processing and machine learning approaches for automatic pavement crack recognition. They 
used advanced image processing techniques (fast local Laplacian filter, Sobel filter, SF, and IP) to extract digital properties from digital images. They benefited from the adaptive boosting classification tree to perform pavement crack recognition tasks. To generate and validate the performance of the adaptive boosting classification tree, a set of image samples consisting of five classes, crocodile cracks, diagonal cracks, longitudinal cracks, no cracks, and cross cracks, were collected. The results of the study revealed that the crack classification accuracy of the proposed approach was approximately $90 \%$. It is considered that the model can be used in the assessment of the pavement condition of the transportation agencies.

\section{Conflicts of Interest}

The guest editors have no conflicts of interest regarding the publication of this special issue.

\section{Acknowledgments}

We would like to thank the authors who have submitted manuscripts to this special issue. We would also like to thank the reviewers for their valuable comments about the submitted paper to this special issue. We hope that the accepted papers for this special issue will lead to the future works related to the advances in optimization techniques. The lead guest editor thanks all the editors for their contribution in reviewing and assigning reviews for the submitted manuscripts. At last, the authors would like to express their gratitude to the journal Advances in Civil Engineering for the opportunity to contribute to an important field of research.

Tayfun Dede

Murat Kankal

Ali Reza Vosoughi

Maksym Grzywinski

Moacir Kripka 


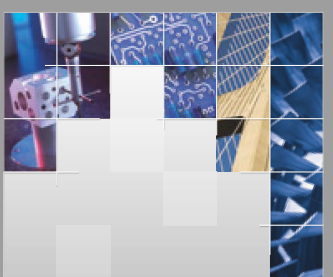

\section{Enfincering}
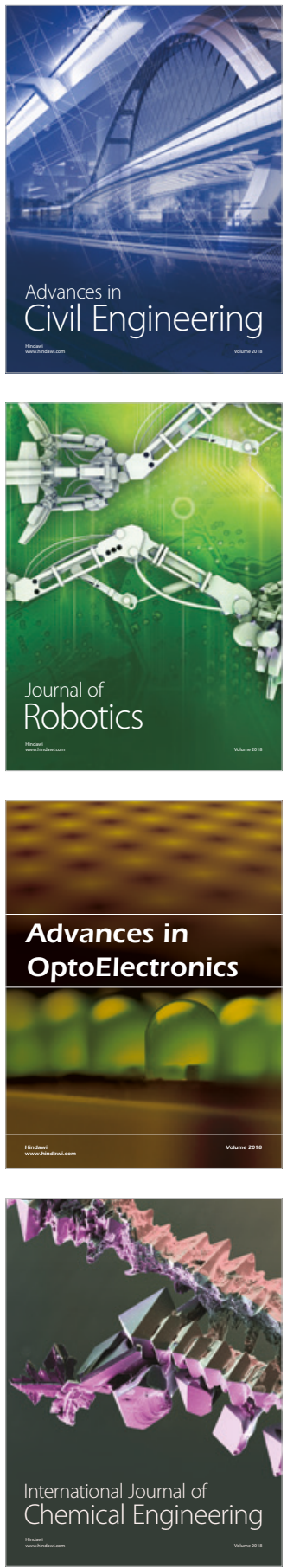

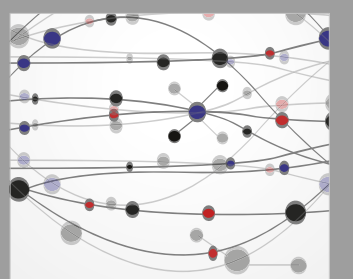

\section{Rotating \\ Machinery}

The Scientific World Journal

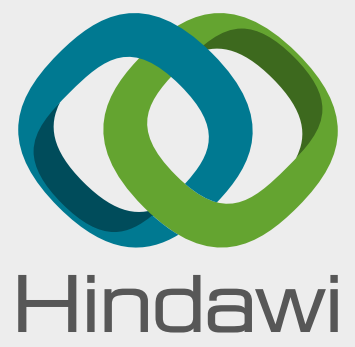

Submit your manuscripts at

www.hindawi.com
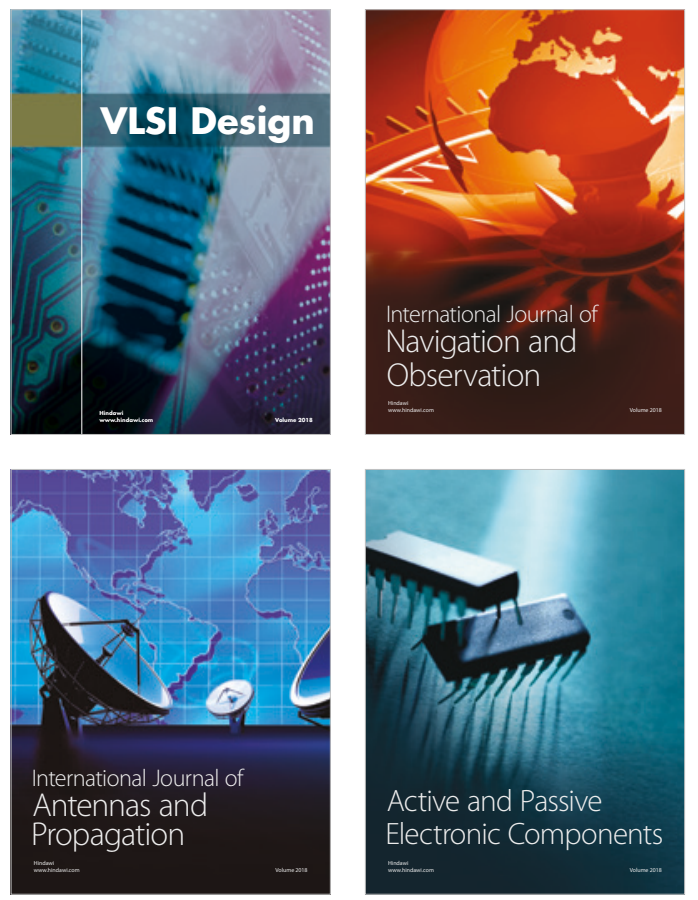
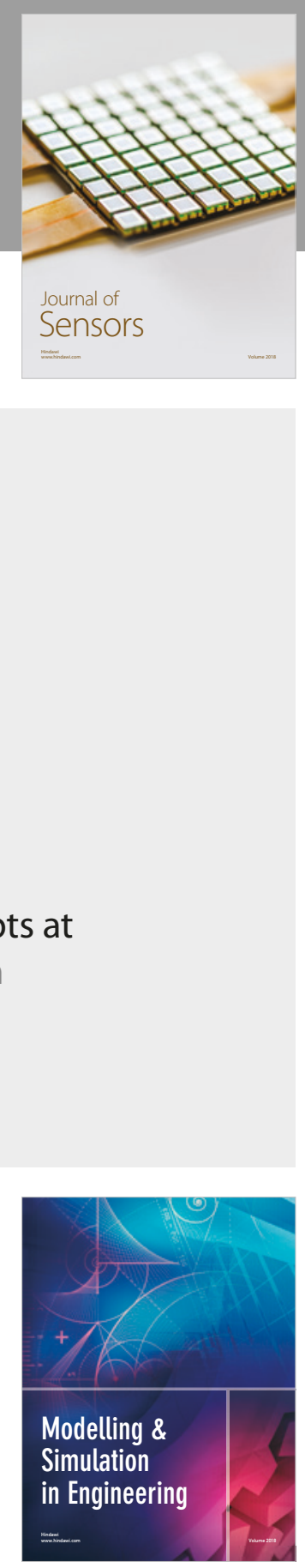

\section{Advances \\ Multimedia}
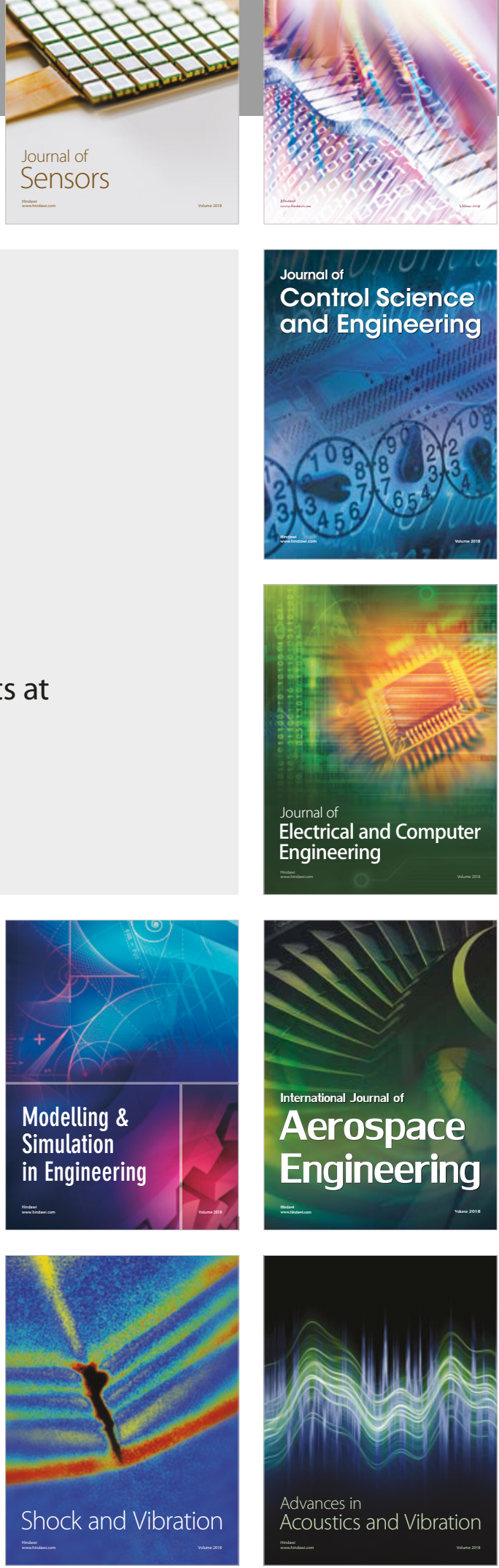